\title{
Effectiveness of Intravenous Ibuprofen On Emergence Agitation in Children Undergoing Tonsillectomy With Propofol Anesthesia: A Double- Blind, Placebo-Controlled, Randomized Study
}

\section{Zhengzheng Gao}

Department of Anesthesiology, Beijing Children's Hospital, Capital Medical University, National Center for Children's Health

Jianmin Zhang ( $\nabla$ sunbrightangel@sina.com )

Department of Anesthesiology, Beijing Children's Hospital, Capital Medical University, National Center for Children's Health

\section{Xiaolu Nie}

Center for Clinical Epidemiology and Evidence-based Medicine, Beijing Children's Hospital, Capital Medical University, National Center for Children's Health

\section{Xiaohuan Cui}

Department of Anesthesiology, Beijing Children's Hospital, Capital Medical University, National Center for Children's Health

\section{Research Article}

Keywords: children, general anesthesia, emergence agitation, ibuprofen, tonsillectomy

Posted Date: November 2nd, 2021

DOI: https://doi.org/10.21203/rs.3.rs-1009839/v1

License: (a) This work is licensed under a Creative Commons Attribution 4.0 International License. Read Full License 


\section{Abstract}

Background: Emergence agitation (EA) has a negative effect on recovery from general anesthesia in children.

Objectives: This study aimed to evaluate the effectiveness of intravenous ibuprofen in reducing the incidence of EA in children.

Methods: This randomized, double-blind, placebo-controlled study analyzed data from patients aged 3-9 years undergoing tonsillectomy with propofol general anesthesia. These patients were randomly assigned to receive either the ibuprofen or the placebo intraoperatively. The primary endpoint was between-group difference in the incidence of EA after surgery. EA was defined as Pediatric Anesthesia Emergence Delirium score $\geq 10$. The secondary endpoint included the associated factors of EA.

Results: Eighty-nine patients were included in the study. Ibuprofen decreased the incidence of EA (8.9\% in the treatment group vs. $34.1 \%$ in the control group; odds ratio [OR], $0.261 ; 95 \%$ confidence interval [Cl], $0.094-0.724 ; P=0.004)$. After the logistic regression analysis, anxiety behavior pre-anesthesia and high pain score after surgery were the risk factors related to $\mathrm{EA}(\mathrm{OR}, 8.07 ; 95 \% \mathrm{Cl}, 1.12-58.07, P=0.038$ and $\mathrm{OR}, 2.78 ; 95 \% \mathrm{Cl}, 1.60-4.82, P<0.001$, respectively). Ibuprofen administration was the protective factor related to $\mathrm{EA}(\mathrm{OR}, 0.05 ; 95 \% \mathrm{Cl}, 0.01-0.67, P=0.023)$.

Conclusions: In our studied cohort, intraoperatively infusing ibuprofen and relieving preoperative anxiety and postoperative pain can significantly reduce the incidence and severity of EA after propofol general anesthesia.

Trial registration: ChiCTR2100045128 (07/04/2021)

\section{Background}

The term emergence agitation (EA) describes an unsettled behavior in children, associated with pain, hunger, thirst, and fear due to the absence of primary caregivers or unfamiliar surroundings. Pediatric EA is an early negative postoperative behavior and consists of two clinical components, the emergence delirium (ED) and postoperative pain, with different trends in the early postoperative period.[1] ED is considered a behavioral disturbance and neurological complication that occurs in children after general anesthesia and described as involuntary agitation with shouting, crying, kicking, absence of eye contact with caregivers, inconsolability, and absence of awareness of the surrounding environment.[2] ED is not exactly equivalent to EA; ED can involve hypoactive signs or mixed forms and hyperactive signs similar to agitation.[3] The terms EA and ED have been used interchangeably in some previous studies.[4, 5] The incidence rate of EA in children induced with general anesthesia ranges from 10-80\%. During EA, children can remove intravenous catheters and drains, damage the surgical site, and injure themselves or the medical staff.[6] The most known risk factors for EA are the presence of an endotracheal tube, preschool age, volatile anesthetics, surgical procedures (ophthalmology and otorhinolaryngology), pre-existing 
behavior, negative behavior on induction, and postoperative pain.[7-13] Although EA can occur in any age group, it is more generally observed in children aged 3-9 years than in other age groups.[14] The incidence rate of EA is $41.4-48.3 \%$ in children undergoing tonsillectomy with propofol and remifentanil anesthesia.[15]

Ibuprofen, a non-steroidal anti-inflammatory drug (NSAID), has been used in patients undergoing tonsillectomy due to its analgesic and opioid-sparing effects; however, in our institution, several anesthesiologists are hesitant to use ibuprofen in tonsillectomies due to their potential for hemorrhage. A recent Cochrane review concluded that NSAIDs did not induce any increase in bleeding that required surgical intervention in pediatric tonsillectomy.[16] Moreover, the American Academy of OtolaryngologyHead and Neck Surgery has recommended ibuprofen as a safe NSAID to reduce postoperative pain in children undergoing tonsillectomy.[17]

We performed total intravenous anesthesia (TIVA) with propofol and remifentanil to detect the effectiveness of intravenous ibuprofen $10 \mathrm{mg} \cdot \mathrm{kg}^{-1}$ in reducing the incidence of EA in children undergoing tonsillectomy with or without adenoidectomy. The secondary endpoint included factors associated with EA in the children.

\section{Methods}

\section{Study design}

This was a single-center, double-blind, placebo-controlled, randomized study. The study protocol was approved by the Ethics Committee of Beijing Children's Hospital, Capital Medical University, National Center for Children's Health, Beijing, China (Chairperson Prof Guojun Zhang) on 23 March 2021 (2021-E015-Y-001). All methods were performed according to relevant guidelines and regulations. The study was conducted at the Beijing Children's Hospital, China, from April to June 2021. Written informed consent was obtained from the parents or guardians before the study procedures were performed. The study was registered with the Chinese Clinical Trial Registry (number: ChiCTR2100045128, date: 07/04/2021). This article adhered to the Consolidated Standards of Reporting Trials guidelines.

\section{Participants}

We enrolled patients aged 3-9 years with an American Society of Anesthesiologists risk score of I-II undergoing elective tonsillectomy with or without adenoidectomy. Children with an abnormal electrolyte balance, previous analgesic treatment, allergy to the study drugs, hepatic, renal, neurological, or neuromuscular disease; craniofacial abnormalities; respiratory or cardiac disease; and patients who chose not to participate were excluded.

\section{Randomization, intervention, and anesthesia management}


Patients were randomly assigned in a 1:1 ratio to receive intravenous ibuprofen or placebo. The treatment allocation order was generated by permuted block randomization with a block size of 6 and was concealed with sequentially numbered sealed envelopes. On the day of the study, a third-party participant opened the envelope and prepared the study drug, which was indistinguishable from each other and marked with a randomization code known only to the participant. Patients and clinical investigators who collected clinical information were blinded to the patient grouping until the final data analysis.

Upon patient arrival in the operating room, conventional monitoring, including electrocardiography, noninvasive blood pressure measurement, pulse oximetry, and bispectral index (BIS) monitoring (BIS Monitor Model A-2000 Aspect Medical Systems Inc., USA), was performed. Anesthesia was administered intravenously with propofol $2 \mathrm{mg} \cdot \mathrm{kg}^{-1}$, fentanyl $2 \mathrm{mcg} \cdot \mathrm{kg}^{-1}$, and cisatracurium $0.1 \mathrm{mg} \cdot \mathrm{kg}^{-1}$ to facilitate endotracheal intubation. After the induction of anesthesia, patients in the treatment group received a dose of $10 \mathrm{mg} \cdot \mathrm{kg}^{-1}$ of intravenous ibuprofen slowly over $15 \mathrm{~min}$, whereas in the control group, a volumematched normal saline infusion was slowly administered. Anesthesia was maintained with propofol and remifentanil, beginning with propofol $10 \mathrm{mg} \cdot \mathrm{kg}^{-1} \cdot \mathrm{h}^{-1}$, followed by an adjustment in dose depending on BIS measurements (40-60), and remifentanil 0.3-0.4 $\mathrm{mcg} \cdot \mathrm{kg}^{-1} \cdot \mathrm{min}^{-1}$ ), with the dose adjusted to analgesic requirements (systolic blood pressure changed within $20 \%$ of baseline values). After the completion of surgery, the patients were transferred to the post-anesthesia care unit (PACU) and extubated once adequate spontaneous breathing was observed. Extubation time was defined as the time interval between discontinuation of anesthetics and extubation.

\section{Data collection and outcomes}

\section{Statistical analysis}

Previous studies have revealed that the incidence rate of EA in pediatric patients undergoing tonsillectomy with propofol anesthesia ranges between $41.4 \%$ and $48.3 \%$ in the PACU. We assumed that the average incidence rate of EA in this study was $45 \%$. Based on our pilot study, we supposed that a reduction in the incidence rate of $>25 \%$ in the ibuprofen group would indicate a significant effect. The sample size was calculated using the tests for a two-proportion design model (one-sided, $a=0.05, \beta=0.8$, $10 \%$ dropout rate), with a group allocation of 1:1 using PASS (NCSS Statistical Software, UT). This resulted in a required sample size of 45 patients per group. Histograms and the Kolmogorov-Smirnov tests were used to assess normality. Continuous variables are expressed as mean \pm standard deviation or median (interquartile interval), as appropriate. To assess the differences between the two groups, the ttest was used for normally distributed continuous variables, whereas the Wilcoxon rank-sum test was used for non-normally distributed continuous variables. For categorical variables, the $\chi 2$ test and Fisher's exact test were used. Secondary analyses were used to build multivariate logistic regression models to assess the association between EA and perioperative variables. Odds ratios (ORs) with $95 \%$ confidence intervals (Cls) for each factor were calculated using logistic regression. Variables with statistically significant values $(P<0.1)$ in the univariate model were entered into the multivariate model. Predictors tested included age, sex, body mass index (BMI), pre-anesthesia score, use of ibuprofen, anesthesia time, 
and extubation time. Model diagnostics were also reported, including the Hosmer-Lemeshow goodnessof-fit test, a receiver operating characteristic (ROC) curve, and the area under the curve (C-index).

Statistical analysis was performed using the International Business Machines Statistical Package for the Social Sciences (SPSS) Statistics version 19.0 (SPSS Inc., Chicago, IL) and GraphPad Prism 9.1 (GraphPad Software Company, San Diego, CA). We selected a significance threshold of $P<0.05$, for comparisons between groups.

\section{Results}

\section{Patient characteristics}

Ninety-three patients were assessed for eligibility. One patient declined to participate in the study, and two patients who did not meet the inclusion criteria were excluded. Finally, 90 patients were randomized. In the control group, one patient only underwent adenoidectomy, depending on the decision of the otolaryngologist during the surgery. Finally, 45 and 44 patients were included in the treatment and control groups, respectively (Figure 1). The mean age and BMI of the patients were 5.0 (4.0-7.5) years and 16.6 $(14.7-18.0) \mathrm{kg} / \mathrm{m}^{2}$, respectively). Furthermore, 47 and 42 patients were men and women, respectively. The demographic and clinical characteristics of the patients are summarized in Table 1 . There were no statistical differences in age, sex, BMI, pre-anesthesia score, anesthesia time, and extubation time between the two groups.

Table 1

Demographic and clinic characteristics of the participants

\begin{tabular}{|llll|}
\hline & Treatment group (n=45) & Control group $(\mathbf{n}=44)$ & $P$ value \\
\hline Male & 24 & 23 & 0.920 \\
\hline Female & 21 & 21 & 0.531 \\
\hline Age (years) & $5.0(4.0-7.5)$ & $5.5(4.0-7.8)$ & 0.783 \\
\hline BMl & $16.6(14.6-18.1)$ & $16.3(14.7-18.0)$ & 0.149 \\
\hline The pre-anesthesia score & & & \\
\hline $1=$ happy & 34 & 27 & 0.799 \\
\hline $2=$ unhappy & 11 & 17 & 0.055 \\
\hline Anesthesia time (min) & $34.0(30.5-47.0)$ & $35.0(30.0-45.0)$ & $14.5(10.0-19.8)$ \\
\hline Extubation time (min) & $17.0(12.0-29.0)$ & & \\
\hline Data are presented as median value (interquartile range), or number of cases. BMl, body mass index. \\
\hline
\end{tabular}




\section{Incidence of emergence agitation and Pediatric Anesthesia Emergence Delirium and Face, Legs, Activity, Cry, Consolability scores}

The PAED and FLACC scores are presented in Table 2. After 15 and 30 min of extubation, the PAED score was lower in the treatment group than in the control group ( $P=0.008$ and $P=0.012$, respectively).

Moreover, the treatment group had a lower FLACC scale score than the control group $(P=0.007$ and $P=$ 0.002 , respectively). The incidence rate of EA at the PACU was significantly lower in the treatment group than in the control group ( $8.9 \%$ vs. $34.1 \%$; OR, $0.261 ; 95 \% \mathrm{Cl}, 0.094-0.724 ; P=0.004)$. The number of patients receiving rescue fentanyl did not differ between the two groups $(22.2 \%$ vs. $34.1 \%$; OR, $0.652 ; 95 \%$ $\mathrm{Cl}, 0.329-1.292 ; P=0.213)$.

Table 2

The emergence agitation and pain score at the PACU

\begin{tabular}{|c|c|c|c|}
\hline & Treatment group $(n=45)$ & Control group $(n=44)$ & $P$ value \\
\hline \multicolumn{4}{|l|}{ PAED scale } \\
\hline PAED 15 & $1.0(0-5.0)$ & $3.5(0.3-10.0)$ & $0.008^{*}$ \\
\hline PAED 30 & $0(0-0.5)$ & $0.5(0-3.0)$ & $0.012^{*}$ \\
\hline \multicolumn{4}{|l|}{ Incidence of EA, $n(\%)$} \\
\hline PAED 15 & $4(8.9)$ & $15(34.1)$ & $0.004^{*}$ \\
\hline PAED 30 & $0(0)$ & $0(0)$ & 1.0 \\
\hline \multicolumn{4}{|l|}{ FLACC scale } \\
\hline FLACC 15 & $1.0(0-3.0)$ & $3.0(1.0-6.0)$ & $0.007^{*}$ \\
\hline FLACC 30 & $0(0-1.0)$ & $1.0(0-2.0)$ & $0.002^{*}$ \\
\hline Number of patients $\mathrm{n}(\%)$ & $10(22.2)$ & $15(34.1)$ & 0.213 \\
\hline \multicolumn{4}{|l|}{ receiving rescue fentanyl } \\
\hline \multicolumn{4}{|c|}{ Data are presented as median (interquartile range), or number of cases. } \\
\hline \multicolumn{4}{|c|}{ PAED, Pediatric Anesthesia Emergence Delirium; FLACC, Face, Legs, Activity, Cry, Consolability } \\
\hline${ }^{*} P<0.05$ & & & \\
\hline
\end{tabular}

\section{Associated factors of EA}


According to the univariate regression analysis, the remaining four predictors (age, pre-anesthesia score, FLACC score [15 min], and ibuprofen administration) were used as independent variables (Table 3).[20] After the multivariate logistic regression analysis, a high pre-anesthesia and FLACC score were the risk factors related to $\mathrm{EA}(\mathrm{OR}, 8.07 ; 95 \% \mathrm{Cl}, 1.12-58.07 ; P=0.038$ and $\mathrm{OR}, 2.78 ; 95 \% \mathrm{Cl}, 1.60-4.82 ; P<0.001$, respectively). Moreover, intravenous ibuprofen administration was a protective factor related to $E A$ at the PACU (OR, $0.05 ; 95 \% \mathrm{Cl}, 0.01-0.67 ; P=0.023$ ) (Figure 2). Pediatric patients with anxiety had 8.07 times higher odds of developing EA compared to patients with calm and controlled anesthesia behavior. Each 1-point increase in the FLACC score was associated with a 1.78-fold increase in EA. Pediatric patients receiving intravenous ibuprofen were $95 \%$ less likely to develop EA than those who did not receive ibuprofen. The Hosmer-Lemeshow goodness-of-fit test was not significant $(P=0.998)$, indicating that the model exhibited a good fit. The predictive ability of the EA risk was examined by generating an ROC curve, and the AUC (C-index) was $0.97(95 \% \mathrm{Cl}, 0.94-1.0)$ (Figure 3). The predictive performance of the EA risk was considered excellent when the $\mathrm{C}$-index was $>0.8$. 
Table 3

Univariable and multivariable model of multivariate logistic regression

\begin{tabular}{|c|c|c|c|c|c|c|}
\hline & \multicolumn{3}{|c|}{ Univariable model } & \multicolumn{3}{|c|}{ Multivariable model } \\
\hline & $B$ value & OR $(95 \% \mathrm{Cl})$ & $P$ value & B value & OR $(95 \% \mathrm{Cl})$ & $P$ value \\
\hline Age (years) & -0.28 & $\begin{array}{l}0.75(0.57 \\
-0.99)\end{array}$ & 0.042 & -0.54 & $\begin{array}{l}0.58(0.31 \\
-1.08)\end{array}$ & 0.086 \\
\hline Sex (female vs male) & 0.01 & $\begin{array}{l}1.01(0.37 \\
-2.79)\end{array}$ & 0.986 & - & - & - \\
\hline BMI & -0.02 & $\begin{array}{l}0.99(0.84 \\
-1.15)\end{array}$ & 0.846 & - & - & - \\
\hline $\begin{array}{l}\text { The pre-anesthesia } \\
\text { score ( } 2 \text { vs } 1)\end{array}$ & 2.42 & $\begin{array}{l}11.20(3.45- \\
36.35)\end{array}$ & $<0.001$ & 2.09 & $\begin{array}{l}8.07(1.12- \\
58.07)\end{array}$ & $0.038^{*}$ \\
\hline $\begin{array}{l}\text { Anesthesia time } \\
\text { (min) }\end{array}$ & -0.01 & $\begin{array}{l}0.99(0.95- \\
1.03)\end{array}$ & 0.647 & - & - & - \\
\hline Extubation time (min) & -0.03 & $\begin{array}{l}0.97(0.92- \\
1.03)\end{array}$ & 0.366 & - & - & - \\
\hline FLACC 15 score & 0.84 & $\begin{array}{l}2.31(1.63 \\
-3.28)\end{array}$ & $<0.001$ & 1.02 & $\begin{array}{l}2.78(1.60- \\
4.82)\end{array}$ & $<0.001^{*}$ \\
\hline $\begin{array}{l}\text { Ibuprofen } \\
\text { administration }\end{array}$ & -1.67 & $\begin{array}{l}0.19(0.06- \\
0.63)\end{array}$ & 0.006 & -3.01 & $\begin{array}{l}0.05(0.01- \\
0.67)\end{array}$ & $0.023^{*}$ \\
\hline \multicolumn{7}{|c|}{$\begin{array}{l}\text { Two logistic regression models were fitted separately. In univariate models, variables with statistically } \\
\text { significant values }(P<0.1) \text { were entered into the final multivariate model. Model diagnostics for final } \\
\text { model: } P \text { value of the Hosmer-Lemeshow goodness-of-fit test }=0.998 \text {, and area under curve }=0.973 \text {. }\end{array}$} \\
\hline \multicolumn{7}{|c|}{ BMI, body mass index; FLACC 15, Face, Legs, Activity, Cry Consolability scale 15 min after extubation } \\
\hline${ }^{*} P<0.05$ & & & & & & \\
\hline
\end{tabular}

\section{Discussion}

In this study, intraoperative infusion of ibuprofen significantly reduced the incidence and severity of EA in pediatric patients aged 3-9 years after tonsillectomy. The patients who received intravenous ibuprofen had lower PAED and FLACC scores than patients who did not receive ibuprofen, indicating that the agitation level was lower than that in the control group, and intravenous ibuprofen more effectively relieved postoperative pain in pediatric patients after tonsillectomy. Interestingly, the number of patients who needed to receive a rescue dose of fentanyl was not significantly different between the two groups. This could be explained by the rescue criteria used in the present study. We set the FLACC score $>3$ (moderate to severe pain) as the standard for rescue fentanyl, resulting in six patients in the treatment group with moderate pain and without EA receiving fentanyl. This is an ethical consideration; we want to relieve the pain of pediatric patients as much as possible. An additional finding of this study was that the 
FLACC score was lower in the treatment group than in the control group at 30 min after extubation. According to the data (Table 2), all patients in both groups had mild pain (FLACC score $\leq 3$ ); thus, this difference in score value had no clinical implications.

Optimal pain management in children undergoing tonsillectomy remains a challenge. NSAIDs are a promising option for the treatment of postoperative pain in children. The opioid-sparing effect of NSAIDs was observed in a previous study.[21] Reducing the use of opioids could lead to a reduction in opioidrelated complications, including vomiting and respiratory system issues. Ibuprofen has anti-inflammatory properties that can limit the inflammatory cascade caused by surgical trauma and reduce the development of postoperative pain. If intravenous ibuprofen is administered before surgery, therapeutic cerebrospinal fluid and blood levels are assessed by the time a patient arrives in the PACU after this short surgery.[22]

EA has been considered as a behavioral and mental disturbance during recovery from general anesthesia that may manifest as agitation, delusion, inconsolability, crying restlessness, disorientation, and cognitive impairment.[23] To predict the incidence of EA in children aged 3-9 years after tonsillectomy with propofol anesthesia, we established a logistic regression prediction model, which showed excellent predictive performance (C-index > 0.8). In this randomized controlled study, some risk factors of EA, which had been revealed through various previous studies, were consistent between patients with or without EA, such as endotracheal tube, operative procedure, and anesthesia (TIVA or inhalation).[24, 25] Among the candidate predictors of EA, the FLACC scores and dose of rescue fentanyl were highly correlated with each other. Thus, the dose of rescue fentanyl was excluded from the univariate model to avoid multicollinearity. In the multivariate model, the risk factors for EA were high pre-anesthesia and FLACC scores. With increasing anxiety before surgery and increasing pain after surgery, the probability of EA increases. Patients who received intraoperative intravenous ibuprofen infusion were less likely to develop EA than those who did not receive ibuprofen infusion. Interestingly, age was not a risk factor in the logistic regression model, possibly because most of the patients in this study were of preschool age, and according to previous studies, preschool age was a risk factor. Moreover, unlike the results of Hino et al., [20] the duration of anesthesia was not a risk factor in our study. They found that the incidence of EA increased only when the duration of anesthesia was $>1 \mathrm{~h}$ in children. In our study, most of the patients were anaesthetized for less than an hour; thus, anesthesia time was not a risk factor in our study.

This study has some limitations. First, this was a single-center study, and the anesthesia time was consistent $(<1 \mathrm{~h})$ at our institution. Therefore, if the duration of anesthesia is prolonged in tonsillectomy at other centers, the predictive power of anesthesia time needs to be reanalyzed. Second, all patients were anaesthetized with propofol and remifentanil; therefore, the outcome of the study cannot be applied to patients anaesthetized with other anesthesia techniques such as volatile anesthetics. Third, the sample size of our study was small, and the OR value of the pre-anesthesia score had a slightly wider confidence interval. Fourth, postoperative follow-up was not performed to further analyze whether intraoperative ibuprofen administration affected postoperative agitation. 


\section{Conclusion}

In summary, the intraoperative infusion of ibuprofen can significantly reduce the incidence and severity of EA after general anesthesia induction in pediatric patients aged 3-9 years undergoing tonsillectomy. Pediatric patients with anxiety before anesthesia induction and more pain after surgery have an increased risk of developing EA.

\section{Abbreviations}

BIS, Bispectral index

$\mathrm{Cl}$, Confidence interval

EA, Emergence agitation

ED, Emergence delirium

NSAID, Non-steroidal anti-inflammatory drug

PACU, Post-anesthesia care unit

PAED, Pediatric Anesthesia Emergence Delirium

ROC, Receiver operating characteristic

SPSS, Statistical Package for the Social Sciences

TIVA, Total intravenous anesthesia

\section{Declarations}

Ethics approval and consent to participate: The study protocol was approved by the Ethics Committee of Beijing Children's Hospital, Capital Medical University, National Center for Children's Health on 23 March 2021 (2021-E-015-Y-001). Written informed consent was obtained from parents or legal guardians before any study procedures were performed. The procedures followed were in accordance with the ethical standards of the responsible committee on human experimentation (institutional or regional) and with the Helsinki Declaration of 1964, as revised in 2000 . The study was registered with the Chinese Clinical Trial Registry under the number ChiCTR2100045128.

Consent for publication: Not applicable.

Availability of data and materials: All data generated or analysed during this study are included in this published article.

Competing interests: The authors declare that they have no competing of interests. 
Funding: No external funding for this manuscript.

Authors' contributions: ZZ. G: Study design, data collection and writing up of the manuscript; JM, Z: Study design, analysis and interpretation of data; XL. N: Study design, data analysis and critically revised the manuscript; $\mathrm{XH}$. C: Data collection and analysis, writing up of the manuscript.

\section{Acknowledgements}

We would like to thank the reviewers for their careful review and critique, which helped us improve the manuscript considerably.

\section{References}

1. Somaini M, Engelhardt T, Fumagalli R, Ingelmo PM. Emergence delirium or pain after anaesthesia-how to distinguish between the two in young children: a retrospective analysis of observational studies. Br J Anaesth. 2016;116(3):377-83.

2. Dahmani S, Delivet $H$, Hilly J. Emergence delirium in children: an update. Curr Opin Anaesthesiol. 2014;27(3):309-15.

3. Meagher DJ, Hanlon D, Mahony E, Casey PR, Trzepacz PT. Relationship between symptoms and motoric subtype of delirium. J Neuropsychiatry Clin Neurosci. 2000;12(1):51-6.

4. Frederick HJ, Wofford K, de Lisle Dear G, Schulman SR. A Randomized Controlled Trial to Determine the Effect of Depth of Anesthesia on Emergence Agitation in Children. Anesth Analg. 2016;122(4):1141-6.

5. Mason KP. Paediatric emergence delirium: a comprehensive review and interpretation of the literature. Br J Anaesth. 2017;118(3):335-43.

6. Kim SY, Kim JM, Lee JH, Song BM, Koo BN. Efficacy of intraoperative dexmedetomidine infusion on emergence agitation and quality of recovery after nasal surgery. $\mathrm{Br} J$ Anaesth. 2013;111(2):222-8.

7. Chandler JR, Myers D, Mehta D, Whyte E, Groberman MK, Montgomery CJ, Ansermino JM. Emergence delirium in children: a randomized trial to compare total intravenous anesthesia with propofol and remifentanil to inhalational sevoflurane anesthesia. Paediatr Anaesth. 2013;23(4):309-15.

8. Beringer RM, Greenwood R, Kilpatrick N. Development and validation of the Pediatric Anesthesia Behavior score--an objective measure of behavior during induction of anesthesia. Paediatr Anaesth. 2014;24(2):196-200.

9. Gooden R, Tennant I, James B, Augier R, Crawford-Sykes A, Ehikhametalor K, Gordon-Strachan G, Harding-Goldson $\mathrm{H}$. [The incidence of emergence delirium and risk factors following sevoflurane use in pediatric patients for day case surgery, Kingston, Jamaica]. Rev Bras Anestesiol. 2014;64(6):4138.

10. Card $E$, Pandharipande $P$, Tomes $C$, et al. Emergence from general anaesthesia and evolution of delirium signs in the post-anaesthesia care unit. Br J Anaesth. 2015;115(3):411-7. 
11. Costi D, Ellwood J, Wallace A, Ahmed S, Waring L, Cyna A. Transition to propofol after sevoflurane anesthesia to prevent emergence agitation: a randomized controlled trial. Paediatr Anaesth. 2015;25(5):517-23.

12. Bedirli N, Akçabay M, Emik U. Tramadol vs dexmedetomidine for emergence agitation control in pediatric patients undergoing adenotonsillectomy with sevoflurane anesthesia: prospective randomized controlled clinical study. BMC Anesthesiol. 2017;17(1):41.

13. Driscoll JN, Bender BM, Archilla CA, Klim CM, Hossain MJ, Nd MG, Wei JL. Comparing incidence of emergence delirium between sevoflurane and desflurane in children following routine otolaryngology procedures. Minerva Anestesiol. 2017;83(4):383-91.

14. Kanaya A. Emergence agitation in children: risk factors, prevention, and treatment. J Anesth. 2016;30(2):261-7.

15. Tsiotou AG, Malisiova A, Kouptsova E, Mavri M, Anagnostopoulou M, Kalliardou E. Dexmedetomidine for the reduction of emergence delirium in children undergoing tonsillectomy with propofol anesthesia: A double-blind, randomized study. Paediatr Anaesth. 2018;28(7):632-8.

16. Cardwell M, Siviter G, Smith A. Non-steroidal anti-inflammatory drugs and perioperative bleeding in paediatric tonsillectomy. Cochrane Database Syst Rev. 2005;(2):CD003591.

17. Baugh RF, Archer SM, Mitchell RB, et al. Clinical practice guideline: tonsillectomy in children. Otolaryngol Head Neck Surg. 2011;144(1 Suppl):S1-30.

18. Merkel SI, Voepel-Lewis T, Shayevitz JR, Malviya S. The FLACC: a behavioral scale for scoring postoperative pain in young children. Pediatr Nurs. 1997;23(3):293-7.

19. Lee YJ, Kim BY, Park JH, Kim SY, Park HY, Do SH. The Effect of Intraoperative Magnesium Sulphate Infusion on Emergence Agitation after Ambulatory Ophthalmic Surgery in Children. J Clin Med. 2020;9(12).

20. Hino M, Mihara T, Miyazaki S, Hijikata T, Miwa T, Goto T, Ka K. Development and Validation of a Risk Scale for Emergence Agitation After General Anesthesia in Children: A Prospective Observational Study. Anesth Analg. 2017;125(2):550-5.

21. Singla N, Rock A, Pavliv L. A multi-center, randomized, double-blind placebo-controlled trial of intravenous-ibuprofen (IV-ibuprofen) for treatment of pain in post-operative orthopedic adult patients. Pain Med. 2010;11(8):1284-93.

22. Vane JR, Botting RM. Mechanism of action of nonsteroidal anti-inflammatory drugs. Am J Med. 1998;104(3A):2S-8S; discussion 21S-22S.

23. Sikich N, Lerman J. Development and psychometric evaluation of the pediatric anesthesia emergence delirium scale. Anesthesiology. 2004;100(5):1138-45.

24. Przybylo HJ, Martini DR, Mazurek AJ, Bracey E, Johnsen L, Coté CJ. Assessing behaviour in children emerging from anaesthesia: can we apply psychiatric diagnostic techniques. Paediatr Anaesth. 2003;13(7):609-16.

25. Kain ZN, Caldwell-Andrews AA, Maranets I, McClain B, Gaal D, Mayes LC, Feng R, Zhang H. Preoperative anxiety and emergence delirium and postoperative maladaptive behaviors. Anesth 
Analg. 2004;99(6):1648-54, table of contents.

\section{Figures}

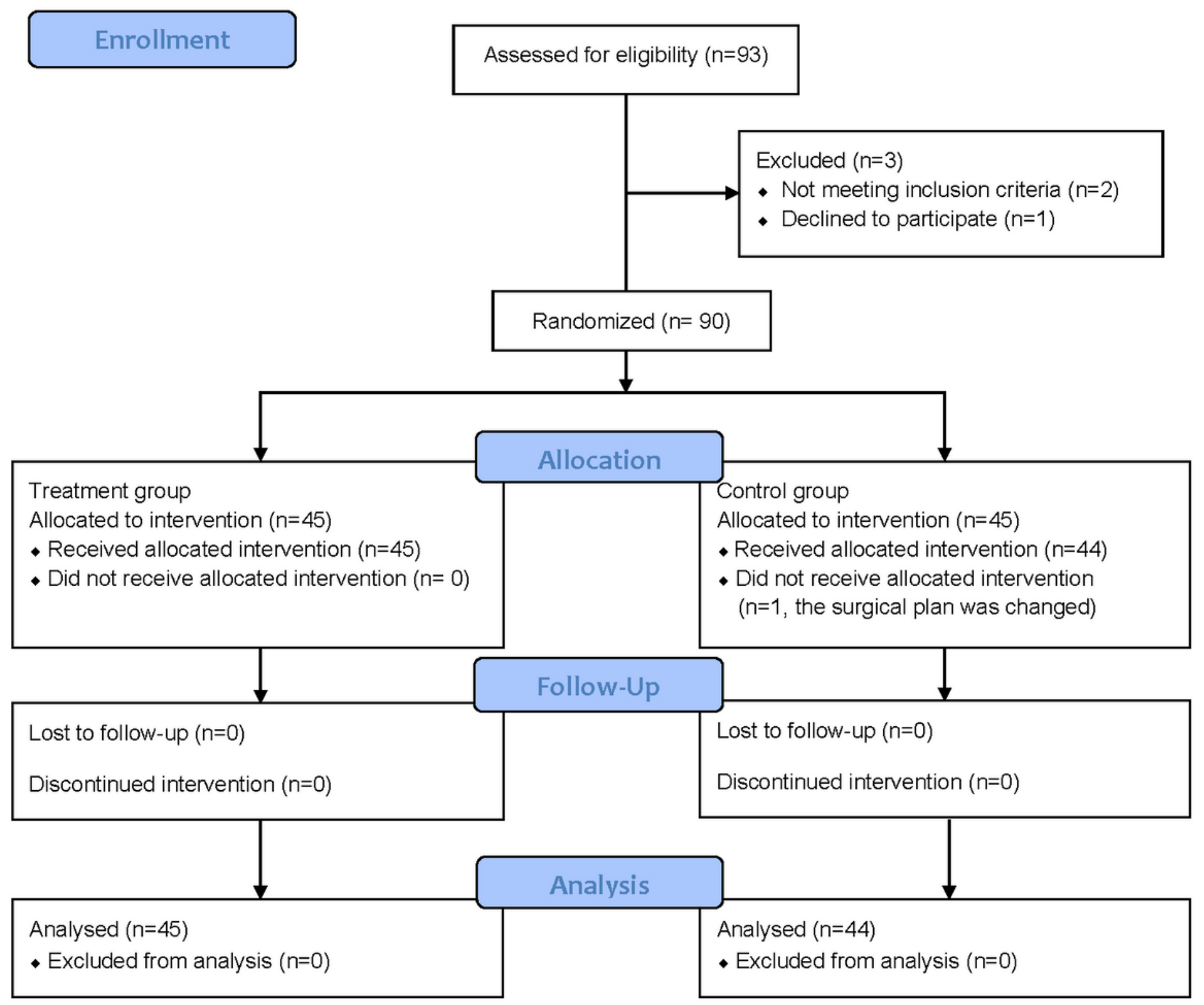

\section{Figure 1}

Flow diagram of the patients in the study. 


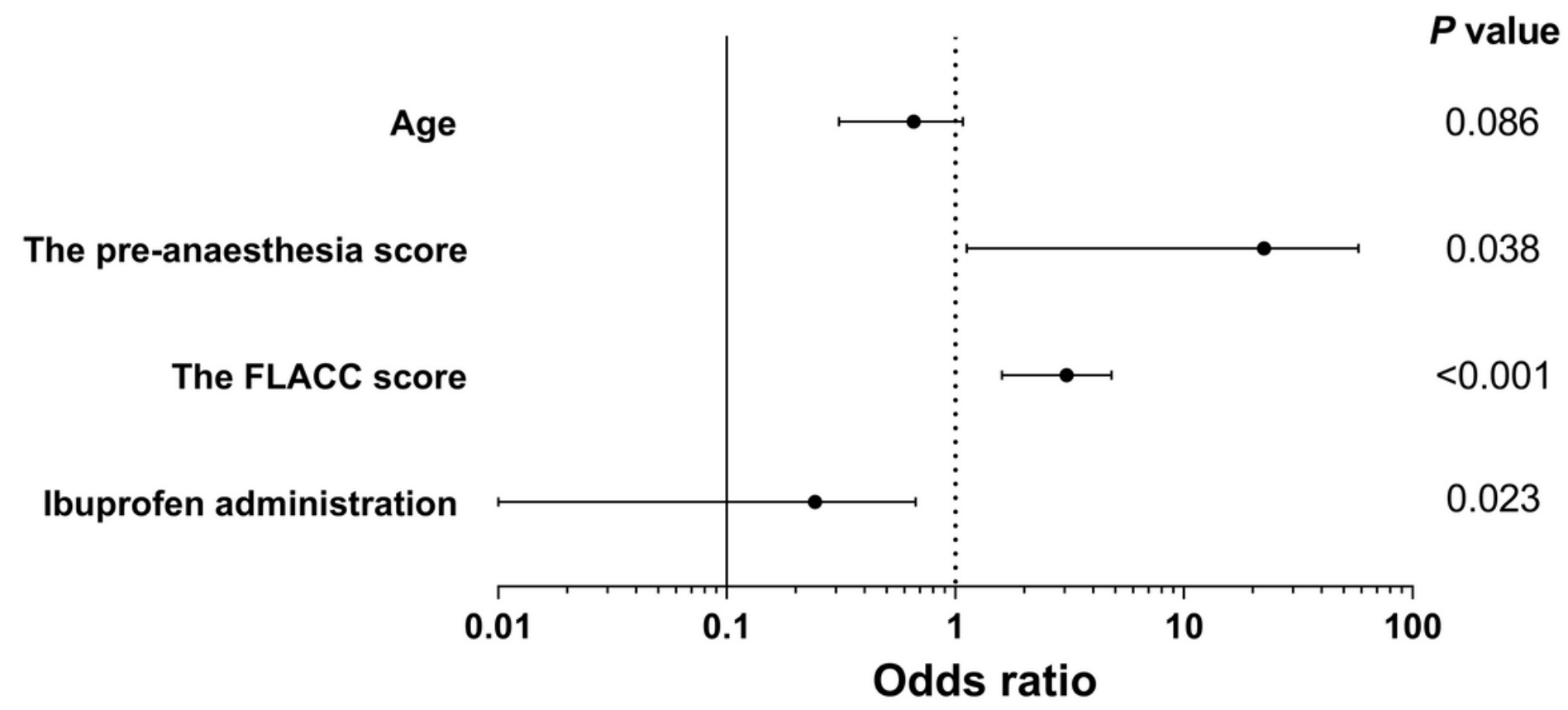

Figure 2

Association between predictors and emergence agitation. 


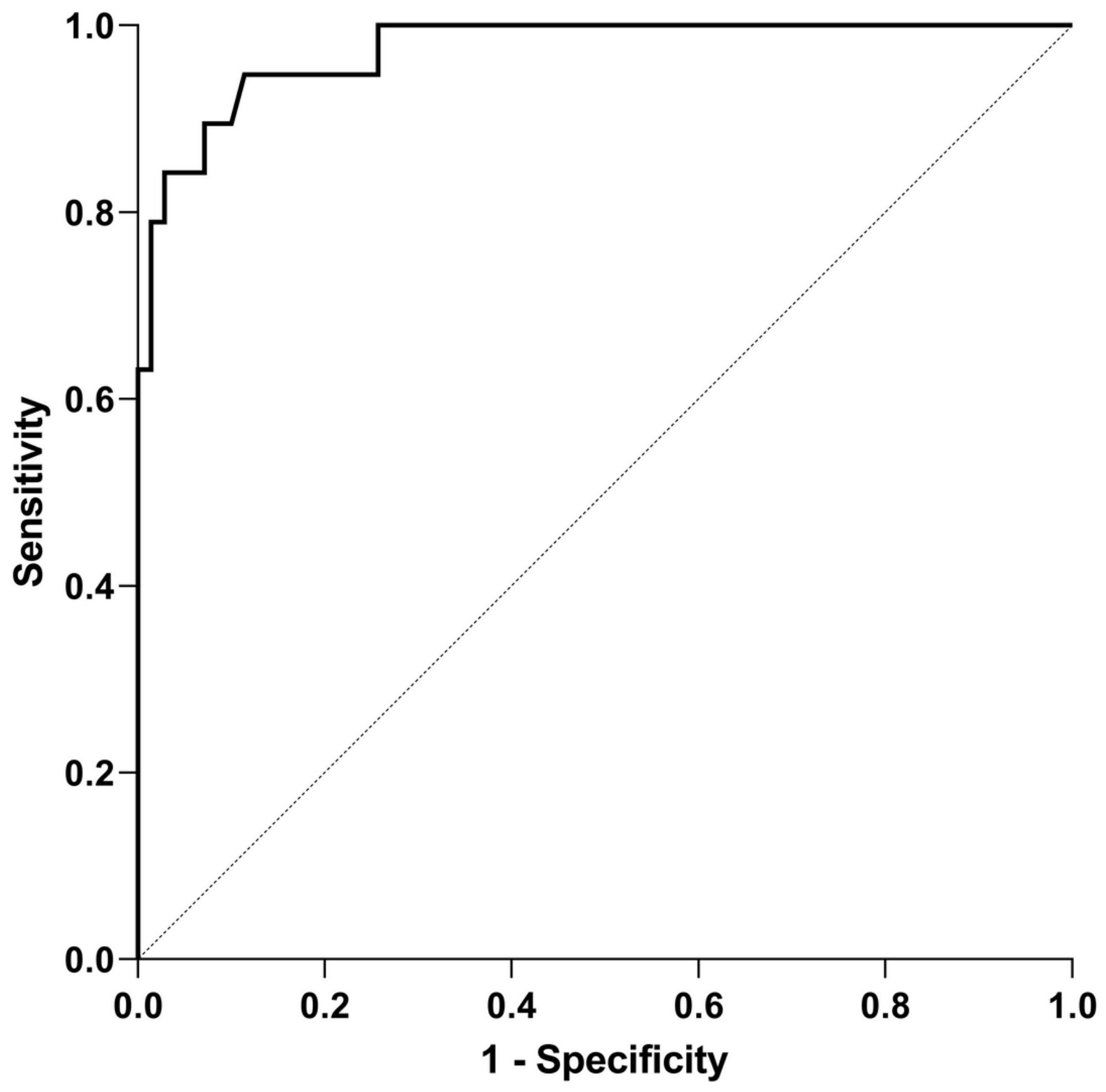

Figure 3

The ROC curve for examining the predictive ability of the final model. The black line indicates the ROC curve. The area under the ROC curve (C-index) was 0.97 (95\% $\mathrm{Cl}, 0.94-1.0)$. ROC, receiver operating characteristic; $\mathrm{Cl}$, confidence interval.

\section{Supplementary Files}

This is a list of supplementary files associated with this preprint. Click to download. 
- SupplementaryAppendixtableS1.docx

- SupplementaryAppendixtableS2.docx 